\title{
Adhesion Pili from Enterotoxigenic Escherichia coli Share Similar Biophysical Properties Despite Their Different Assembly Pathways
}

Narges Mortezaei, ${ }^{1}$ Chelsea R. Epler, ${ }^{2}$ Paul P. Shao, ${ }^{2}$ Mariam Shirdel, ${ }^{1}$ Bhupender Singh, ${ }^{1,3}$ Annette McVeigh, ${ }^{4}$ Bernt Eric Uhlin, ${ }^{3}$ Stephen J. Savarino, ${ }^{4,5}$ Magnus Andersson ${ }^{1}$ Esther Bullitt ${ }^{2}$

${ }^{1}$ Department of Physics, Umeå University, SE-901 87 Umeå, Sweden.

${ }^{2}$ Department of Physiology \& Biophysics, Boston Univ. School of Medicine, Boston, MA 02118, USA. 3The Laboratory for Molecular Infection Medicine Sweden (MIMS) and Department of Molecular Biology, Umeå University, SE-901 87 Umeå, Sweden.

${ }^{4}$ Enteric Diseases Department, Infectious Diseases Directorate, Naval Medical Research Center, Silver Spring, MD 20910, USA.

${ }^{5}$ Department of Pediatrics, Uniformed Services University of the Health Sciences, Bethesda, MD 20814, USA.

Enterotoxigenic Escherichia coli (ETEC) cause diarrhea, enabled by the expression of different adhesion pili. These long, thin, helical filaments, also called fimbriae, share structural similarities, but exhibit varied force requirements for unwinding the filaments into thin fibrillae. The ability of adhesion pili to unwind reduces the shear forces acting on bacteria during intestinal transit, thereby facilitating the sustained adhesion that is necessary for initiation of disease.

There is limited genetic similarity between pilins from distinct adhesion pili,. Those in the gastrointestinal (GI) tract can be equally similar to pilins on bacteria that colonize the GI or urinary tracts. Additional diversity arises from pilus assembly via one of two pathways, either the chaperoneusher pathway (CUP), or the alternate chaperone pathway (ACP), independent of the environmental niche of the bacteria. For example, different ETEC express and assemble CFA/I pili via ACP, and CS20 pili via CUP.

Our work [1,2] shows that the structures of these two ETEC pili, CFA/I and CS20, share similar structural properties, with diameters of 7-8 nm, lengths of 1-3 $\mu \mathrm{m}, 3.2$ subunits per turn of the helix, and 0.8 or $0.9 \mathrm{~nm}$ rise per subunit, respectively. A three-dimensional reconstruction of CS20 pili from electron cryomicroscopy data has been computed and compared to both CFA/I pili and to P-pili expressed on E. coli that cause urinary tract infections (UTIs). Results show that the inclination of subunits in pili from the GI pathogens is similar, but CUP assembled pili are more similar with respect to the number of subunit-subunit contacts between layers of the helix (Figure 1).

Physical properties of CS20 pili were measured using force measuring optical tweezers. The unwinding force of CS20 was assessed to $15+/-1 \mathrm{pN}$. (Figure 2) These results are compared to forces measured for other pili expressed on bacteria in the GI and urinary tracts. First, when the subunit orientation is steeper and the number of contact points is the same, the unwinding force required is higher. Second, when the subunit orientation is the same and the number of contact points is reduced, the unwinding force is lower.

In addition, dynamic force measurements are used to examine the velocity at which filament unwinding requires increased force, defining its 'corner velocity'. We see a broad distribution of corner velocities 
that appear to be inversely related to the force of unwinding. Thus, higher corner velocities are measured on pili with lower unwinding forces.

In summary, new data on CS20 pili are providing structural and biophysical details that allow comparative analysis between adhesion pili that are essential virulence factors on pathogenic bacteria that cause diarrheal disease or urinary tract infections.

\section{References:}

[1] Mortezaei et al., 2015. Molecular Microbiology 95(1), 116-126.

[2] Li et al., 2009. Proc. Natl. Acad. Sci 106(26), 10793-10798.

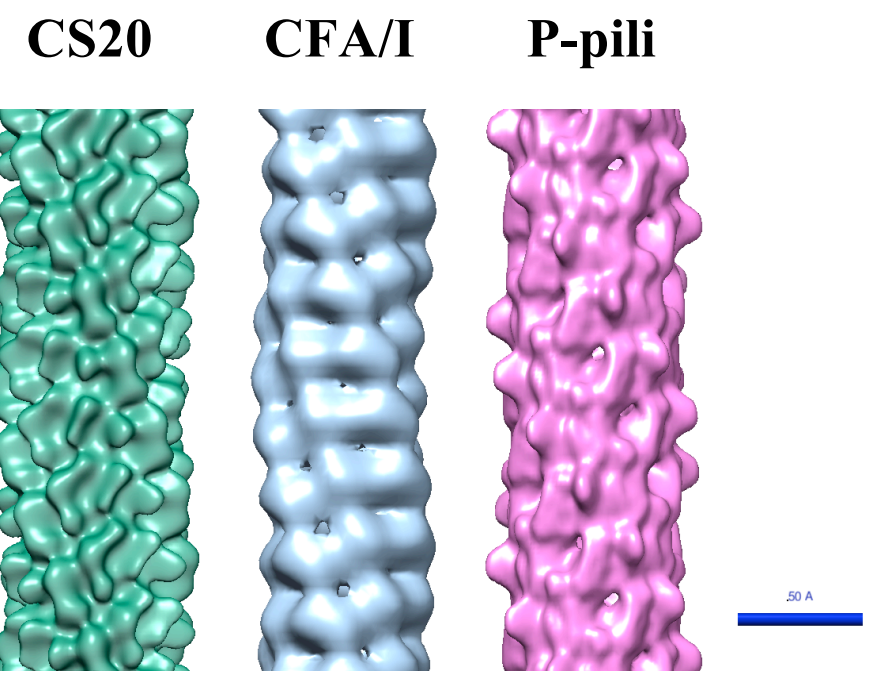

Figure 1. Three-dimensional helical reconstructions of adhesion pili from pathogenic bacteria: cryoEM reconstruction of CS20 pili; negative stain reconstruction of CFA/I pili, and cryoEM reconstruction of P-pili. CS20 and CFA/I are expressed on bacteria that cause diarrheal disease, and P-pili are expressed on bacteria that cause urinary tract infections. Magnification bar, $5 \mathrm{~nm}$.

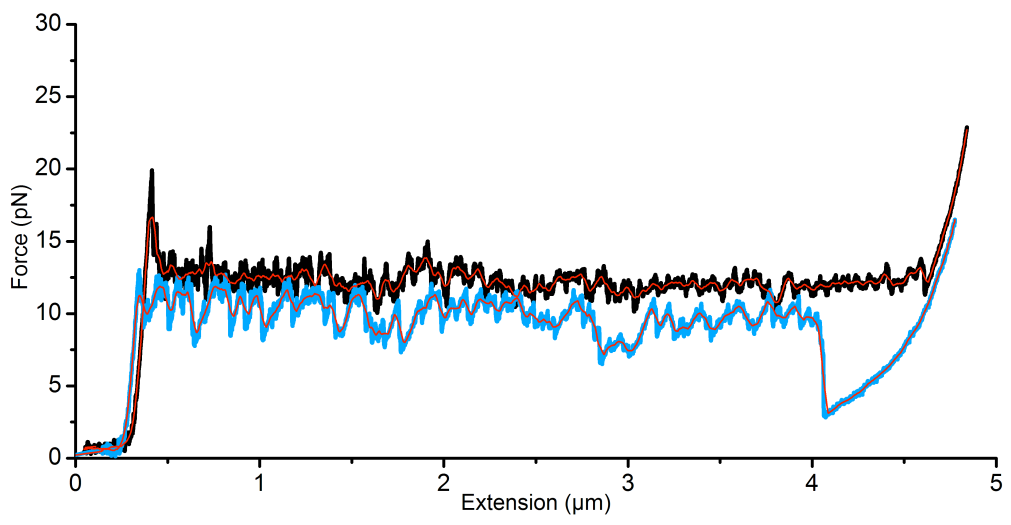

Figure 2. Force spectroscopy data showing that steady-state CS20 pilus unwinding occurs at $15 \mathrm{pN}$ force (black curve). The pilus can be re-wound (blue curve) after a dip in force corresponding to nucleation required for re-winding. 